\title{
A tapetal ablation transgene induces stable male sterility and slows field growth in Populus
}

\author{
Estefania Elorriaga - Richard Meilan • Cathleen Ma - Jeffrey S. Skinner • \\ Elizabeth Etherington • Amy Brunner • Steven H. Strauss
}

Received: 20 March 2014 / Revised: 18 July 2014 / Accepted: 18 July 2014 / Published online: 13 August 2014

(C) The Author(s) 2014. This article is published with open access at Springerlink.com

\begin{abstract}
The field performance of genetic containment technologies-considered important for certain uses of transgenic trees in forestry-is poorly known. We tested the efficiency of a barnase gene driven by the TA29 tapetum-dominant promoter for influencing growth rate and inducing male sterility in a field trial of transgenic hybrid poplar (Populus tremula $\times$ Populus tremuloides). When the growth of 18 transgenic insertion events with the sterility transgene were compared to non-transgenic controls after two growing seasons, they grew $40 \%$ more slowly in stem volume, and all but one
\end{abstract}

Communicated by R. Sederoff

Electronic supplementary material The online version of this article (doi:10.1007/s11295-014-0781-6) contains supplementary material, which is available to authorized users.

E. Elorriaga · R. Meilan · C. Ma · J. S. Skinner · E. Etherington ·

A. Brunner $\cdot$ S. H. Strauss $(\square)$

Department of Forest Ecosystems and Society, Oregon State

University, Corvallis, OR 97331, USA

e-mail: Steve.Strauss@oregonstate.edu

Present Address:

R. Meilan

Department of Forestry and Natural Resources, Purdue University,

West Lafayette, IN 47907, USA

Present Address:

J. S. Skinner

Bayer CropScience Vegetable Seeds, Crop Genomics, 279 Cousteau

Place, Suite 200, Davis, CA 95618, USA

Present Address:

E. Etherington

College of Agricultural Sciences, Oregon State University, Corvallis, OR 97331, USA

Present Address:

A. Brunner

Department of Forest Resources and Environmental Conservation,

Virginia Tech University, Blacksburg, VA 24061, USA transgenic event grew significantly more slowly than the control. In contrast, when we compared the growth of transgenic trees containing four kinds of $\beta$-glucuronidase (GUS) reporter gene constructs to non-transgenic trees - all of which had been produced using the same transformation method and poplar clone and grown at the same field site - there were no statistically significant differences in growth after three growing seasons. In 2 years where gross pollen release from catkins was monitored and found to be abundant in the control, no pollen was visible in the transgenic trees; microscopy suggested the cause was tapetal collapse and revealed the presence of a very few normal-sized pollen grains of unknown viability. In two additional years when viable, well-formed pollen was microscopically documented in controls, and no pollen could be observed in any transgenic trees. We conclude that this construct resulted in robust and possibly complete male sterility that was stable over 4 years in the field.

Keywords Populus · Barnase · TA29 promoter · Pollen · Risk assessment · Forest biotechnology · Genetic containment . Genetic engineering

\section{Introduction}

Genetically engineered (GE) trees in field trials have shown improved wood quality; faster growth; and markedly improved insect, disease, herbicide, and abiotic-stress resistance (Harfouche et al. 2011; Hinchee et al. 2009). However, regulations and substantial market barriers hinder research progress and commercial applications. A major obstacle to application of GE trees is concern over transgene dispersal in the environment (Strauss et al. 2009a, b). Although most pollen from wind-pollinated woody plants falls close to its point of release, a minority can travel from hundreds of meters to several kilometers. For example, paternity analyses done in 
two northwestern Populus trichocarpa populations, one in western Oregon and another in eastern Oregon, revealed that one third to one half of the fertilizing pollen originated from beyond 1 and $10 \mathrm{~km}$, respectively (DiFazio et al. 2012; Slavov et al. 2009). When sexually compatible, pollen can fertilize the abundant wild and feral populations of poplars that are common in many temperate-zone regions (James et al. 1998). A minority of seed-abetted by animal-, water-, or stormassociated dispersal - can also travel large distances and establish in the wild. Due to this potential for wide dispersal and because the possible ecological effects of novel genes in the wild are difficult to predict with confidence, there has been long-term interest in the development of containment methods to prevent or strongly mitigate transgene dispersal.

There are several major genetic containment strategies that have been discussed for forest trees (reviewed in Brunner et al. 2007; Vining et al. 2012). These include fitness reduction, ablation, transgene excision, and floral gene suppression at the RNA or protein levels. Ablation methods have been most widely studied and in the case of floral sterility rely on cellor tissue-dominant promoters to drive expression of a cytotoxin gene to destroy tissues essential for gamete development. Previous studies using the pTA29::BARNASE construct in alfalfa (Medicago sativa, Rosellini et al. 2001), oilseed mustard (Brassica juncea, Jagannath et al. 2001), oilseed rape (Brassica napus, Mariani et al. 1990), tobacco (Nicotiana tabacum, Mariani et al. 1990), and wheat (Triticum aestivum, De Block et al. 1997) demonstrated that this construct is effective in inducing male sterility by disrupting the development of tapetal cells. Jagannath et al. (2001) also found that the Arabidopsis tapetal promoter A9 was highly effective; $94 \%$ of the transformants with the tobacco TA29 promoter and $87 \%$ of the transformants with the A9 promoter showed stable male sterility, and none of the plants produced seed by selfing (i.e., reverted to being fertile). Mariani et al. (1990) found that 106 out of 115 transformed tobacco events showed stable male sterility. The 14 transgenic lines of wheat studied by De Block et al. (1997) had one to three copies of the barnase gene, and all but one showed stable and complete male sterility that was inherited in offspring of a varietal hybrid. The constructs BpMADS1::BARNASE (Lemmetyinen et al. 2001, 2004) and BpFULL1::BARNASE (Lännenpää et al. 2005) produced male sterility in Arabidopsis, tobacco (N. tabacum) (Lännenpää et al. 2005; Lemmetyinen et al. 2001), and silver birch (Betula pendula) (Lännenpää et al. 2005; Lemmetyinen et al. 2004). The employed promoters were derived from the birch BPMADS1 gene (homologous to the Arabidopsis gene SEPALLATA3, previously known as $A G L 19$ ) and the birch BPFRUITFULLLIKE1 gene. Zhang et al. (2012) showed that a male conedominant promoter from Monterey pine (Pinus radiata), PrMC2, fused to an attenuated version of barnase gave complete and stable male sterility in multi-year field trials of a pine hybrid (Pinus rigida $\times$ Pinus taeda) and a eucalypt hybrid (Eucalyptus grandis $\times$ Eucalyptus urophylla).

There have been several reports of vegetative abnormalities when the barnase gene was employed for sterility. In the greenhouse studies of silver birch (Lännenpää et al. 2005; Lemmetyinen et al. 2004), transformants were bushy, short, and grew more weakly than the control. Jagannath et al. (2001), studying Brassica, found that constructs with the strong constitutive cauliflower mosaic virus (CaMV) $35 \mathrm{~S}$ promoter driving the selectable marker gene showed more vegetative abnormalities than transformants with a weaker promoter driving the selectable marker gene. They inferred this to be a result of $35 \mathrm{~S}$ enhancer effects causing vegetative expression of barnase. Thus, it is important to carefully evaluate vegetative growth in transgenic plants containing barnase-based ablation transgenes.

We report that a TA29::BARNASE transgene was highly effective at inducing male sterility in poplar and did so in the field over several years, but it also caused significant growth retardation. These results show that sterility transgenes can be highly effective at mitigating transgene dispersal in poplar, but that further technology development and testing are required to establish methods for imparting female sterility and reducing undesired effects on growth.

\section{Materials and methods}

\section{Gene constructs}

Sterility Hybrid poplars were transformed with the binary plasmid pTTM8 provided by Plant Genetic Systems (Gent, Belgium). The vector (described in Li et al. 2007) contained three transgenes within its transfer DNA (T-DNA), including $B A R N A S E$, neomycin phosphotransferase II (NPTII), which provides resistance to kanamycin, and bar (selectable marker for glufosinate ammonium herbicide resistance). The $B A R N A$ $S E$ gene was derived from Bacillus amyloliquefaciens (Mariani et al. 1990) and encodes a ribonuclease.

Reporter To evaluate whether transformation affected growth rate, we produced a number of transgenic trees that lacked the $B A R N A S E$ gene and contained only the $\beta$-glucuronidase (GUS) reporter gene and the selectable marker gene for kanamycin resistance. Between 1998 and 1999, ten transgenic events were produced via the same Agrobacterium tumefaciens transformation methods for each of the four GUS transgene constructs: PTD::GUS (abbreviated 3PG), EnACT11::GUS (3A11G), EnACT2::GUS (3A2G), and En35S::GUS (3SG) (Electronic Supplementary Material (ESM), Table S1). The promoter from the PTD gene is expressed in a floral-predominant manner (Sheppard et al. 2000; Skinner et al. 2003), while the other three promoters 
(EnACT11, EnACT2, and En35S) allow for approximately constitutive expression (An et al. 1996; Huang et al. 1997). In brief, constructs En35S::GUS::E9, EnACT11::GUS::E9, and EnACT2::GUS::E9 were generated using pMON10547 as the binary vector backbone (ESM, Table S1). The vector pMON10547 contains two copies of the 35S promoter enhancer region, the $35 \mathrm{~S}$ promoter basal region, a multi-cloning site (MCS), and the E9 transcriptional terminator. Construct En35S::GUS::E9 was generated by adding the GUS reporter gene behind the constitutive $35 \mathrm{~S}$ promoter. Both actin promoters, EnACT11 and EnACT2, were amplified from Arabidopsis thaliana ecotype Columbia based on the corresponding actin2 (AT3G18780) and actin11 (AT3G12110) gene sequences, using primers with flanking restriction enzyme adaptor sites. The ACT2 promoter region was amplified using primers ACT2.001 (ESM, Table S2) and ACT2.002 (ESM, Table S2), and the ACT11 promoter region using primers ACT11.001 (ESM, Table S2) and ACT11.002 (ESM, Table S2). Amplicons were subcloned into the polymerase chain reaction (PCR) cloning vector pCR-TOPO2.1 and confirmed by Sanger sequencing. The cloned promoters were excised and subcloned into the binary vector to replace the $35 \mathrm{~S}$ basal promoter region (i.e., the -90 fragment of Benfey and Chua 1990) while retaining the upstream double $35 \mathrm{~S}$ promoter enhancer regions to generate constructs EnACT2::GUS::E9 and EnACT11::GUS::E9. The tandem $35 \mathrm{~S}$ promoter enhancer regions were thus fused upstream of the respective actin promoter to augment the endogenous constitutive actin regulatory and basal promoter regions. Sanger sequencing was performed to confirm the correct assembly of the respective promoter GUS reporter gene fusion constructs EnACT2::GUS::E9 and EnACT11::GUS::E9.

\section{Micropropagation}

Sterile in vitro young microcuttings (shoot tips and micronodes) of male hybrid aspen genotype (INRA 353-38, Populus tremula $\times$ Populus tremuloides) were propagated on hormonefree, half-strength Murashige and Skoog (MS) medium (Murashige and Skoog 1962). Plants grew on these media for 4-6 weeks, and then, micropropagation was repeated three to five times until enough plants for field tests were produced. They were then were transplanted to soil and acclimated in the greenhouse and field prior to planting in field trials.

\section{Transformation}

Sterile in vitro cultures of genotype 353-38 were used for all transformations. Inter-nodes and leaf disks, in admixture, were used as explants for cocultivation. All transformation and regeneration were essentially as described in Filichkin et al. (2006), using A. tumefaciens strain C58/pMP90 (GV3101). For the sterility construct, 18 independent transgenic events were generated, and each transgenic event was micropropagated to produce three ramets for field testing (except for event 2, which had four). There were nine nontransgenic wild-type controls propagated in the same way. For the reporter gene field study, ten events were produced using each construct and multiplied and readied for the field essentially the same as for the sterility field study.

\section{Transgene confirmation}

Genomic DNA was isolated from leaf tissue using the DNeasy Plant Mini Kit (Qiagen, Valencia, CA) following the manufacturer's instructions. All transgenic plants were confirmed by PCR against one or more target genes within the T-DNA. We used non-transgenic, in vitro grown trees produced at the same time as negative PCR controls. The NPTII and BARNA $S E$ genes were amplified from the male-sterile trees (ESM, Table S2), and the GUS gene was amplified in the reporter trees (ESM, Table S2); negative controls, using template from a non-transgenic control, were also included during all PCR analyses. For simplicity, genotype designations have been recorded in this manuscript as follows (format is "original code/manuscript code"): 7/1, 43/2, 15/3, 102/4, 2/5, P/6, 58/ 7, 29/8, 39/9, 8/10, 38/11, 28/12, 16/13, 40/14, 9/15, 30/16, $41 / 17$, and $63 / 18$.

\section{Field layout}

The reporter and sterility field trials were planted adjacent to one another in a field site near the Willamette River in Benton County, OR. The sterility trial was planted in September 1995 and the reporter trial in September 2000; both trials were irrigated in the first and second growing season after planting.

The sterility trial was planted in a randomized block design with three blocks; each block contained a single ramet of each transgenic event (except for block 1 which had two ramets for event 2), and each block had three ramets for each control type. The reporter trial was planted in a completely randomized design with ten transgenic events for each construct (except 3PG, which had three ramets) and two wild-type controls for each construct (except for the 3PG construct, which had four), for a total ten control trees. The spacing was $3 \mathrm{~m}$ between rows and $1.5 \mathrm{~m}$ within rows. As described above, for the reporter trial, all transgenic events started with two ramets (except for event 3PG96, which had three). However, by 2003, one event each in $3 \mathrm{~A} 2 \mathrm{G}, 3 \mathrm{PG}$, and $3 \mathrm{SG}$, and one control ramet (out of the 10), was lost due to animal damage or unknown causes. We conducted a one-way ANOVA with constructs (including control as a "construct") as the main effect to test if mean volume index varied among constructs for the years 2001 and 2003. The volume index of the different construct-associated control groups was not 
significantly different (ESM, Tables S3 and S4), so all controls were pooled for analysis.

\section{Tree growth measurements}

Tree height and/or stem diameter at $1.5 \mathrm{~m}$ above ground were measured for both trials. Where height and diameter were both available, they were used to calculate a volume index (height $\times$ diameter $^{2}$ ) for each tree. Volume index data for the sterility trial is presented after two growing seasons, approximately at the onset of visible inter-tree competition. Data for the reporter trial is presented for growth after one and three growing seasons; the latter was at the onset of inter-tree competition.

\section{Catkin collection and measurements}

Flowering was studied only in the sterility trial, where catkins were collected every year from 2006 to 2009 starting just prior to the onset of pollen release, and then, additional catkins were collected for approximately 1 month or until no new catkins could be seen opening on trees. Prior to the onset of flowering, two out of the three original blocks had to be removed due to other demands for that land. Catkins were placed in coolers with ice immediately after collection. Because the branches bearing catkins were approximately 10 to $30 \mathrm{~m}$ above the ground, pole pruners and hydraulic lifts were used to facilitate sampling (Fig. 1). During the collection period in 2009, we also took data on catkin morphology (length, weight, and curvature). To quantify a visible difference in catkin curvature, we measured six visibly representative catkins, one from the control and one from five different transgenic events, during the March 17th collection, using ImageJ freeware (Schneider et al. 2012). The angle made by a line projected from the catkin base with that from the catkin tip was used to approximate curvature.

\section{Pollen dehiscence and assessment}

After catkin length and weight were measured, they were placed in a refrigerator until they completed development and began to release pollen. Full pollen release was observed after transfer to Petri dishes kept at room temperature $\left(\sim 20{ }^{\circ} \mathrm{C}\right)$. Catkins were shaken to aid in release of pollen, and the pollen was then diluted in water for microscopic quantification. The numbers of well-formed (i.e., normal and uniform size and shape) grains were counted under a dissecting microscope with a hemocytometer (ESM, Figs. S1 and S2). For each sample, seven hemocytometer squares were counted. To estimate pollen viability in 2008 and 2009, we used 2,2,5-triphenyl tetrazolium chloride (TTC) (Cook and Stanley 1960); only control pollen was stained because the transgenic trees did not produce any detectable pollen. Photos of catkins in Petri dishes were taken with an Axio camera model AxioCam ICc 1 and a ZEISS Stemi SV11 Apo stereomicroscope, and the photos were examined using Carl Zeiss Vision/Axiovision software (release 4.8.1, November 2009). Images of catkins from transgenic events and controls releasing pollen in Petri dishes were taken for all the years of study (2006-2009). Images of some catkins were previously published in review papers by Brunner et al. (2007) and Vining et al. (2012) and thus not shown here.

\section{Anther microscopy}

To observe anther structure, catkins were placed in a formalinacetic acid-alcohol (FAA) fixative solution and vacuum infiltrated for $1-2 \mathrm{~h}$ and then stored at $4{ }^{\circ} \mathrm{C}$ in the dark. For the histological images taken for anthers collected in 2006 and 2009, samples were fixed, dehydrated, embedded in glycol GMA methacrylate plastic, sectioned, and mounted on slides. Sections were stained in $0.5 \%$ toluidine blue $\mathrm{O}$ in citrate buffer. Photographs were taken using a DFC 290 camera with a Leica DM5000 microscope at both $\times 10$ and $\times 25$ magnifications and analyzed using the Leica Application Suite software on the camera. Histology images from 2006 were published previously and not presented here (Brunner et al. 2007; Dalton et al. 2013).

\section{Quantitative analyses}

Statistical analyses were conducted using the R statistical computer language and environment version 3.0.1 ( $\mathrm{R}$ Core Team 2013) using the $R$ packages lsmeans (Lenth 2013), multcomp (Hothorn et al. 2008), and nlme (Pinheiro et al. 2013). Because of heteroscedasticity observed after inspection of residuals, all models were fit using generalized least squares (Hothorn et al. 2008; Lenth 2013; Pinheiro et al. 2013) with variances allowed to differ among constructs or events. Graphical checks of the residuals then showed that variances were approximately uniform and normal in distribution. For the sterility trial, we conducted a two-way ANOVA with blocks and events (including controls) as main effects to test if mean volume index varied significantly. Following ANOVA, a Dunnett's multiple comparison test was used to compare the mean volume index of each transgenic event against the control. For the reporter trial, we carried out a one-way ANOVA to test if mean volume index varied among constructs or controls for the years 2001 and 2003 separately. We also conducted a one-way ANOVA for each construct to test if mean volume index varied among events within a construct. Following ANOVA, a Dunnett's multiple comparison test was used to compare the mean volume index of lines from each construct against the control.

In 2009, catkins were collected from events 7, 9, 12, 14, 17, and control trees on March 10th and March 17th to measure their length and weight (no catkins were collected from event 17 on March 10th). During the first collection, the numbers of catkins collected were $47,37,54,36$, and 60 , corresponding to 
Fig. 1 Field trial during early growth and catkin collection. a Photographed in July 1997, two growing seasons after planting. Note the person $(\sim 1.8 \mathrm{~m})$ just visible within the trees (center bottom). $\mathbf{b}$ The trees in November 1998, after the third growing season after planting. c Collecting catkins with a lift while using a pole pruner during March 2009. d Collecting catkins by hand with a Swedish ladder and a pole pruner in February 2007

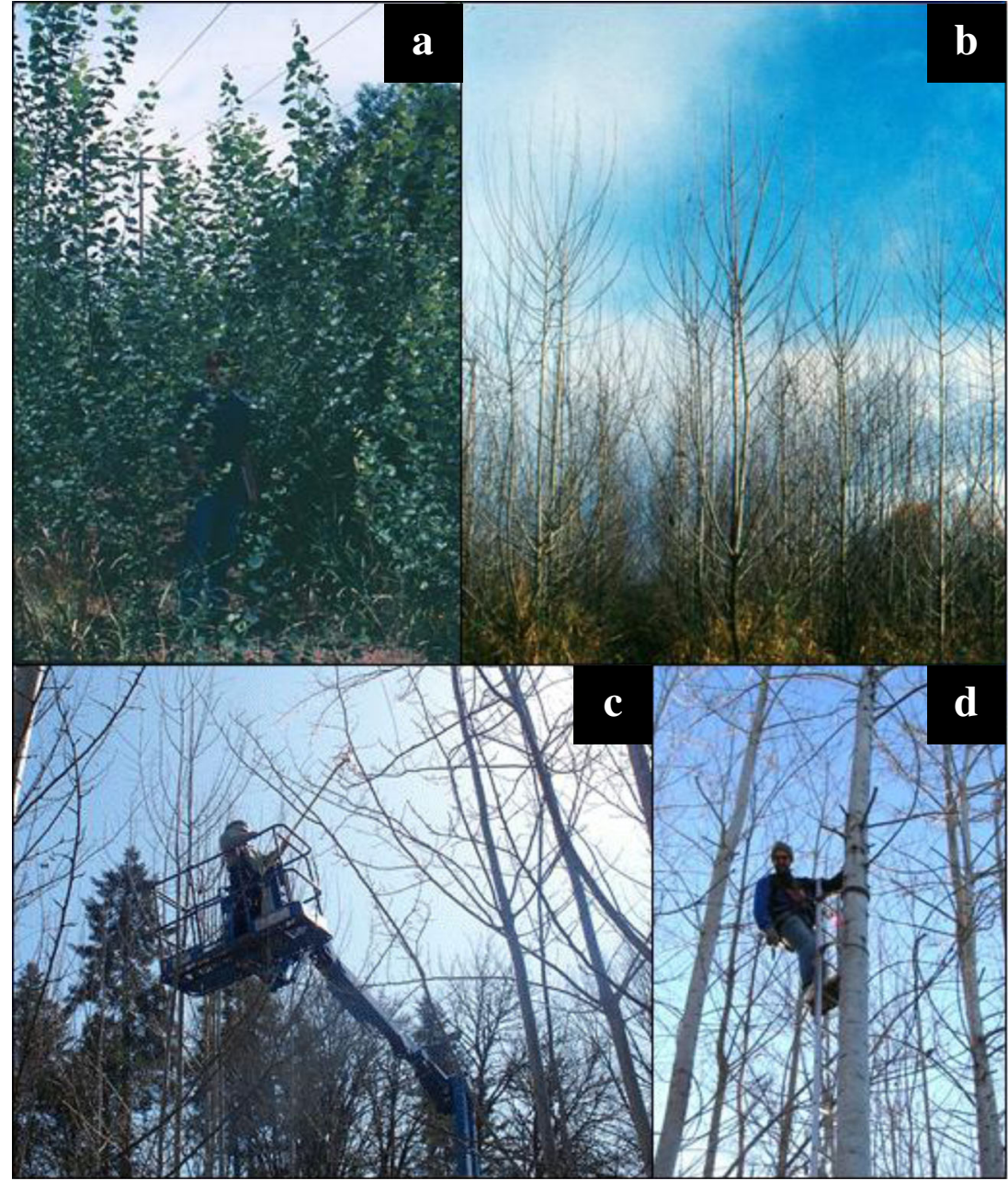

events $7,9,12,14$, and control, respectively. On the second collection, the numbers collected were $6,31,14,13,9$, and 35 , corresponding to events $7,9,12,14,17$, and control, respectively. Mean length was calculated by averaging the length of all the catkins from a single event or control. A Dunnett's multiple comparison test was used to compare the mean catkin length of each transgenic event against that of the control for each collection date. Mean weight was determined by averaging the total weight of all the catkins belonging to an event by the number of catkins. A single-sample Student's $t$ test was used to compare the mean catkin weight of each transgenic event against that of the control for each collection date.

\section{Results}

Slowed early growth of transformants with a barnase transgene

Statistical analysis of early growth in the sterility trial showed that blocks and events were significant sources of variance $\left(F_{2,46}=52, p<0.0001\right.$; ESM, Table S5). All of the transgenic events showed lower mean volume indexes than the control (Fig. 2), and all but one of the 18 events were statistically different (smaller) than the control based on Dunnett's test (Fig. 2; ESM, Table S6). On average, the sterility transgenic events cumulatively grew $40 \%$ more slowly than the control trees over the two seasons of the growth trial.

Absence of evidence for growth impairment due to transformation

Because expression of the GUS reporter gene in transgenic plants is widely known to be essentially free of pleiotropic effects on plant phenotypes (e.g., Gilissen et al. 1998), we employed a population of these plants for helping to assess whether the growth loss we observed in our sterility trial could have been due to transformation itself. In the reporter trial, the mean volume indexes of the transgenic constructs were not significantly different from each other or the controls in 2001 (one year of growth) $\left(F_{4,36}=0.212, p=0.930\right.$; Fig. 3; ESM, Table S7) or in 2003 (3 years of growth) $\left(F_{4,36}=0.263, p=\right.$ 0.900 ; ESM, Table S8). The lack of differences among constructs was also revealed by Dunnett's test for both 2001 and 


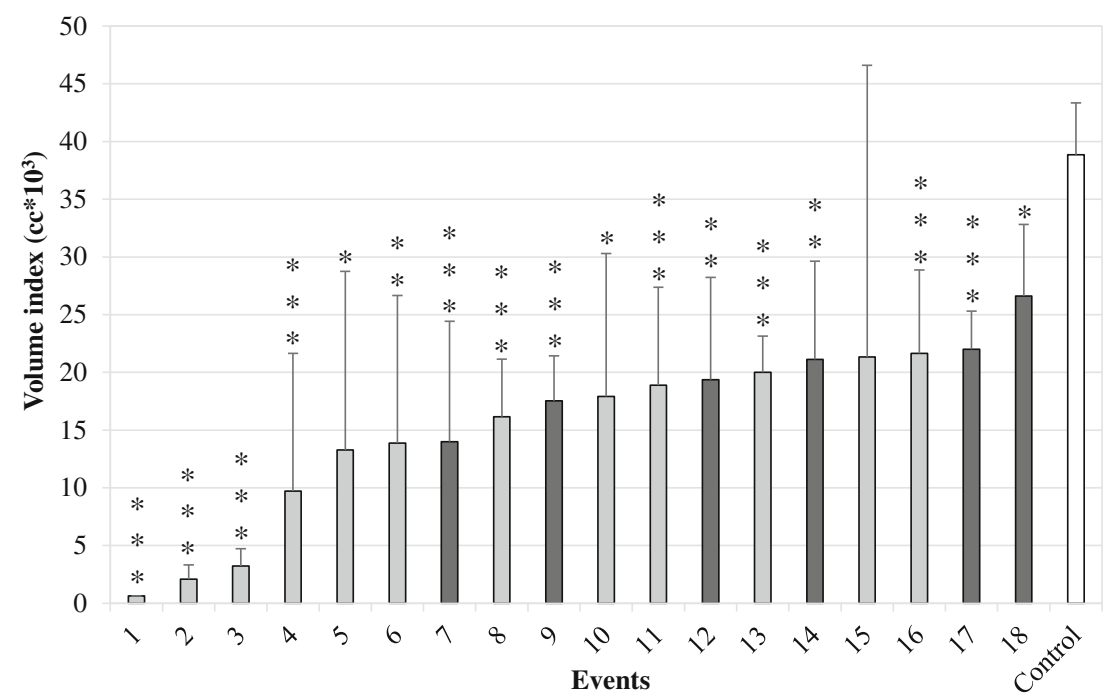

Fig. 2 Transgenic trees showed reduced growth when compared to nontransgenic control. The heights and diameters of all the trees were measured in fall 1997, two growing seasons after planting. Each bar identifies an individual gene insertion event or control. The brackets represent $95 \%$ confidence intervals. The asterisks indicate whether the volume index of the specific event was significantly different than the control based on a Dunnett's test $\left(* * * p<0.001,{ }^{* *} p<0.01\right.$, and ${ }^{*} p<0.05$; all rounded up). The events with darker bars were also studied for their pollen sterility; $c c$ cubic centimeters

an average of 73,000 and 85,000 pollen grains per catkin in years 2006 and 2007, respectively, whereas only a few possible pollen grains (based on similarity in size and shape to wild-type pollen) were observed from transgenic trees in 2006 (Brunner et al. 2007) and 2007 (ESM, Fig. S1). In 2008 and 2009, no pollen, viable or otherwise, was macroscopically or microscopically detected from the transgenic trees, though it was abundantly produced by the control trees (ESM, Fig. S2). For the years 2008 and 2009, the control trees released approximately 775,000 and $2,700,000$ pollen grains per catkin, respectively. The abundant pollen that was produced on the control catkins was also highly visible when whole catkins with mature anthers were examined; the control catkins had swollen anthers, but the anthers of transgenics were shrunken (Fig. 5). 0.116 ); however, for the second collection, when catkin were more fully mature, the weight of the control catkins was significantly lower than that of the transgenic catkins $(p=0.009)$ (ESM, Fig. S4). When we measured the angle projected from the two tips of the catkin toward one another, the transgenic catkins were distinctly curved $\left(91^{\circ} \pm 10.3^{\circ}\right)$, whereas control catkins all appeared perfectly straight $\left(180^{\circ}\right)($ ESM, Fig. S5; Table S15).

Absence of pollen during visual inspection of transgenic catkins

Based on visual inspection of whole Petri dishes after manual agitation, we found that none of the transgenic trees released significant amounts of pollen during any of the years of study (Fig. 4). After microscopic inspection, control trees released
Transgenic anthers showed a collapsed tapetum and absence of pollen

In 2009, we selected catkins from one of the sterile lines (event 12) to study in further detail the structure and morphology of the anther sacs, including the tapetum. Microscopic analyses showed that transgenic anthers had significant developmental abnormalities compared to control anthers (Fig. 6). The tapetum of the transgenic anthers appears to have collapsed, and no pollen grains were observed inside the pollen sacs. It was difficult to differentiate the tapetum from the endothecium, but it appears that the tapetum was completely ablated, and the endothecium appeared thicker than in wild type. 
Fig. 3 Reporter and nontransgenic trees grew at similar rate. The volume index of each transgenic construct was not significantly different from the controls (all $p>0.60$, see Tables S7 and S8). Brackets represent $95 \%$ confidence intervals. Darker bars show data from 2001, and lighter bars show data from 2003; $c c$ cubic centimeters

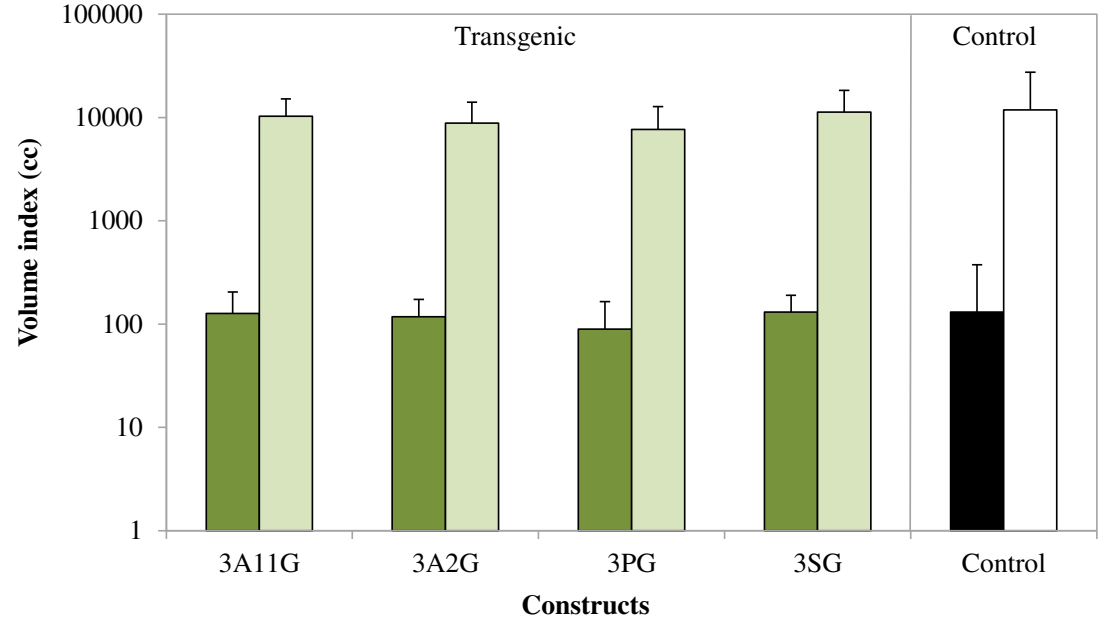

\section{Discussion}

Based on macroscopic inspection of dehiscing catkins, all of the flowering transgenic trees were male-sterile in all 4 years of study. In the 2 years where pollen viability was also determined and analyzed microscopically, only the control produced viable pollen; no pollen was detected from the transgenics. In the review paper by Brunner et al. (2007), preliminary data based on hemocytometer counts were presented that showed extremely little pollen production in 2006; of six events studied, two produced no detectable pollen, and the other four events produced a mean of six pollen grains per catkin, consistent with the very rare grains we observed in 2007 (ESM, Fig. S2). However, viability was not determined in either 2006 or 2007. Thus, it remains unclear if transgenic trees in this study could produce any viable pollen.

Our results showed strong and consistent, but possibly not absolute, male sterility. This finding is similar to several other studies. Lemmetyinen et al. (2004) saw no pollen in transgenic birch. Similarly, no pollen was observed on any transformed tobacco plants (Mariani et al. 1990) nor on transgenic wheat (De Block et al. 1997). Moreover, none of the wheat produced seed from selfing; seed was only produced when cross-pollination was performed. Studying Brassica, Jagannath et al. (2001) found that all of the transformed lines lacked pollen, and none of the plants produced seed by selfing or reverted to being fertile. However, Jagannath et al. (2001) also produced semisterile plants, and they noted that many of them had both sterile and fertile anthers, the former of which eventually reverted to being fully fertile. Rosellini et al. (2001) noted "traces" of pollen in three out of five transformed alfalfa lines, while two lines showed no pollen at all. The three transgenic pollinating lines were selfed and produced just a few seeds; however, the control produced greater than 6fold more seed than the transgenic lines.
Fig. 4 Absence of visible pollen release from transgenic catkins. a, c Show control catkins with released pollen. $\mathbf{b}, \mathbf{d}$ Show transgenic catkins without visible released pollen. b Catkins correspond to event 17. d Catkins correspond to event 14. a, b Catkins were collected in 2007. c, d Catkins were collected in 2009. Images of catkins from 2006 and 2008 were previously published in Brunner et al. (2007) and Vining et al. (2012)

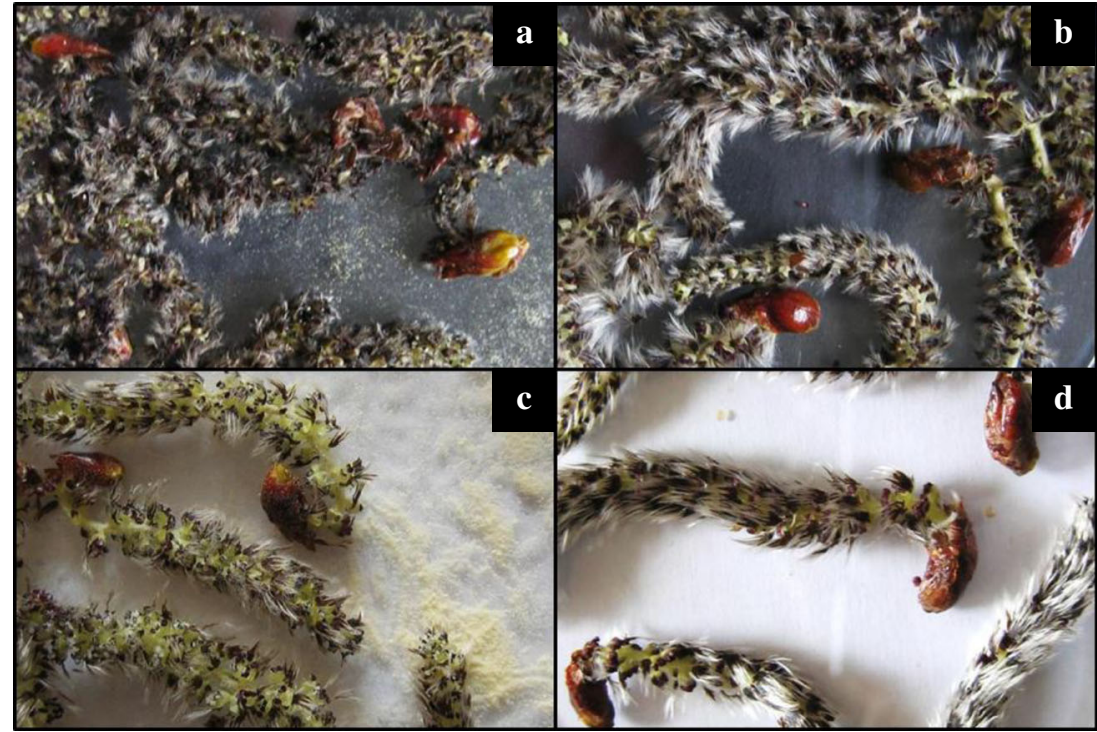




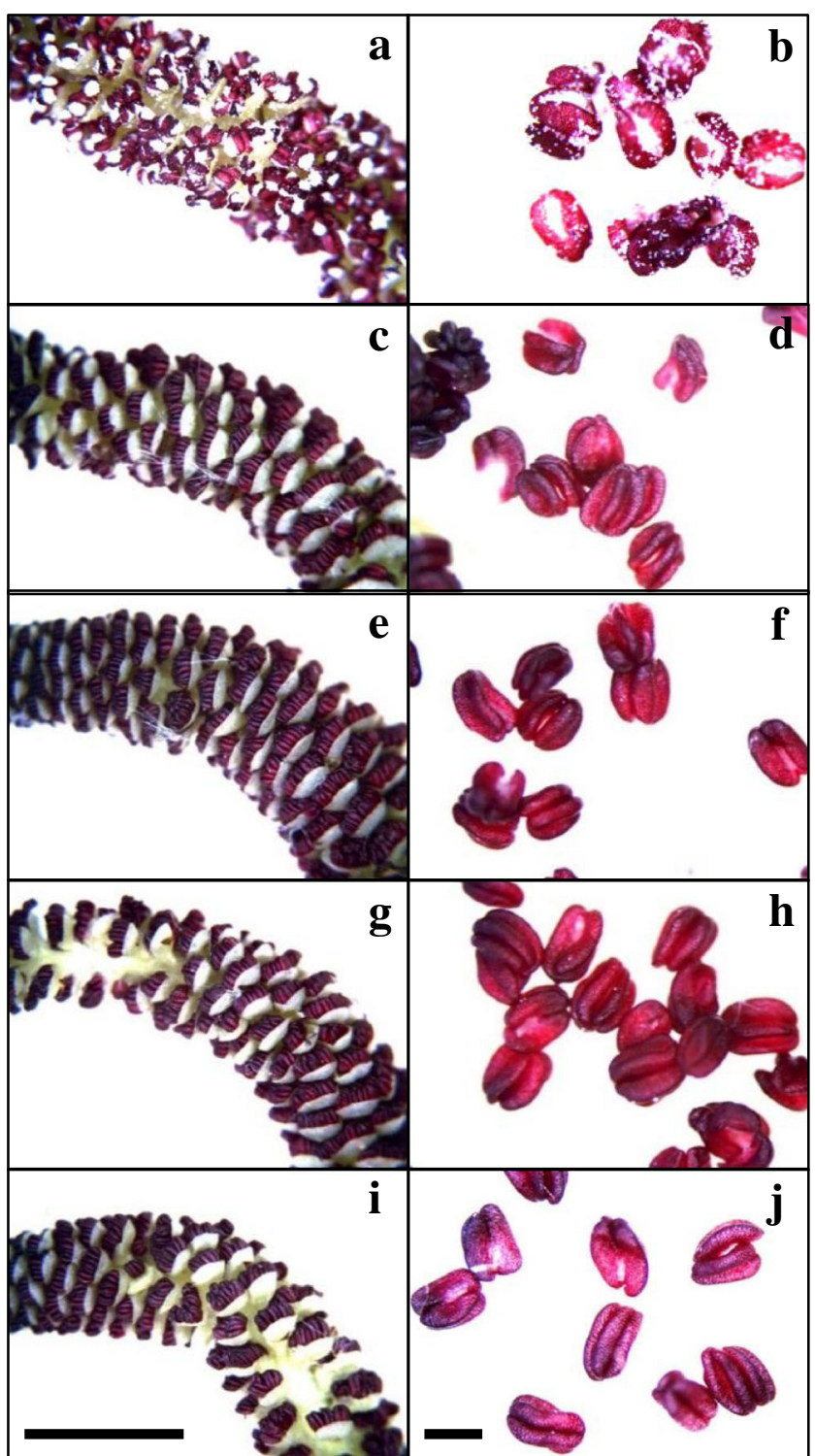

Fig. 5 Transgenic catkins lacked visible pollen. a, b Catkin and stamens are from a control tree. $\mathbf{c}, \mathbf{d}$ Catkin and stamens are from transgenic event 12. e, f Event 9. g, h Event 14. i, j Transgenic event 7. The black bar in the catkin image $\mathbf{i}$ corresponds to $1 \mathrm{~cm}$, and the black bar in the stamen image j corresponds to $1 \mathrm{~mm}$. Photos were taken during March 2009. Histology photos from 2006 can be found in Brunner et al. 2007 and Dalton et al. 2013

In addition to pollen sterility, we found alterations in catkin morphology in the transgenic trees. The catkins from the transformants were smaller and curved when compared to the control catkins (ESM, Figs. S3 and S5). In one collection, we were surprised to find that the transgenic catkins were heavier than the control catkins (ESM, Fig. S4). We hypothesize that this is because the control catkins were weighed after most of their pollen had been released. The control catkins were significantly longer than the transgenic catkins (ESM, Fig. S3); it is unlikely that predehiscence catkins would also be lighter. Male-sterile Brassica transformants also had smaller flowers than controls (Jagannath et al. 2001).
The pollen sacs in our transformants were collapsed, with the tapetum and pollen grains absent. Similar results were reported by Mariani et al. (1990), De Block et al. (1997), and Rosellini et al. (2001) in tobacco, wheat, and alfalfa, respectively. In transgenic alfalfa, deterioration of the tapetum was evident during premiotic development (Rosellini et al. 2001).

Because of its potent and nearly indiscriminant degradation of cellular RNA, barnase is toxic in both prokaryotes and eukaryotes (Ulyanova et al. 2011). All of the transgenic trees we studied had inferior growth to the control trees. This growth difference had also been briefly reported earlier in a preliminary report from our laboratory (Skinner et al. 2000). The GUS enzyme is one of the most widely used reporter genes in transgenic plants, partly because it is not toxic to transformed cells (Gilissen et al. 1998; Miki and McHugh 2004). Transgenic GUS plants have shown no growth effects (Gilissen et al. 1998) or pleiotropic changes in gene expression (Ouakfaoui and Miki 2005) when compared to nontransgenic control plants. Moreover, in a randomized greenhouse study, Lemmetyinen et al. (2001) saw no significant difference in growth between their non-transgenic control line and a transgenic line containing BpMADS5::GUS.

Based on the lack of evidence for growth effects of GUS reporter constructs in the literature and the current results where GUS transgenic trees did not differ from nontransgenic trees in volumetric growth, the data implicate the barnase cytotoxin transgene as the probable cause of the slowed early tree growth seen in the male-sterile transgenic poplars. Reduced growth associated with barnase expression was also seen in greenhouse studies of silver birch (Lännenpää et al. 2005; Lemmetyinen et al. 2004). In the study with the BpFULL1 promoter, one third of their 12 non-flowering lines showed severely reduced growth and small narrow leaves (Lännenpää et al. 2005), and in the study with the BPMADS 1 promoter, 38 of the 45 non-flowering transformants studied were weaker, shorter, and showed abnormal dichotomous branching. Vegetative impairment was seen in all Brassica transformants (Jagannath et al. 2001) where the CaMV 35S promoter drove expression of the bar selectable marker gene in a construct containing the TA29 promoter driving expression of the barnase gene. The authors attributed the morphological abnormalities in their transformants to the unintended expression of barnase in vegetative tissues associated with $35 \mathrm{~S}$ enhancers, as has been reported in other studies (Yoo et al. 2005). Interestingly, in the Jagannath et al. study (2001), no morphological abnormalities were seen in transformants with a 5 -kb spacer between the $35 \mathrm{~S}$ promoter and the barnase gene driven by the TA29 promoter. Our barnase construct, however, did not include the $35 \mathrm{~S}$ promoter. Instead, the nopaline synthase gene promoter (NOSp) drove the NPTII gene and the promoter from the ribulose-1,5-bisphosphate carboxylase small subunit 
Fig. 6 Collapsed tapetum and absence of pollen in transgenic anther sacs. a, c Show control anther sac with pollen. $\mathbf{b}, \mathbf{d}$ Anther sac with collapsed tapetum belonging to transgenic event 12. For both control and transgenic, the black scale bar in b corresponds to $2.5 \mathrm{~mm}$ and the black scale bar in $\mathbf{d}$ to $1 \mathrm{~mm}$. All images were from collections made during February 2009. E epidermis, $E n$ endothecium, $T$ tapetum, $P G$ pollen grain, $P S$ pollen sac

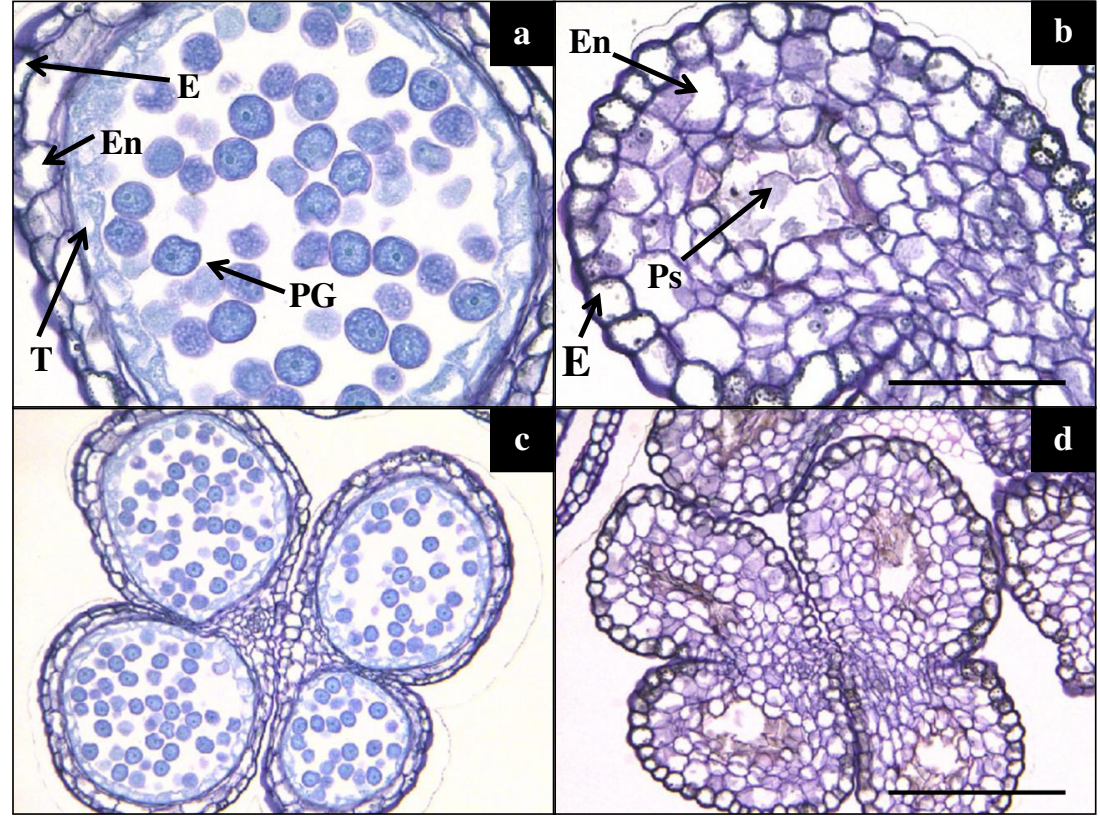

$(r b c \mathrm{~S})$ gene from Arabidopsis, atS1A, drove the bar gene. Both of these promoters are generally considered to be expressed in numerous tissue types; however, the NOS promoter has shown significantly less expression (at least 30fold) of marker genes compared to the $35 \mathrm{~S}$ promoter in both petunia and moss (Horstmann et al. 2004; Sanders et al. 1987). The alfalfa $r b c \mathrm{~S}$ promoter also showed much weaker activity than the $35 \mathrm{~S}$ promoter in young leaves, old leaves, shoot tips, and nodules of alfalfa (Samac et al. 2004). Nonetheless, we believe that the NOSp, together with the effects of "random" transgene integration, was a likely cause of unintended barnase expression in vegetative tissues and thus growth inhibition. It is also likely that the TA29 promoter has imperfect tissue fidelity, especially in the taxonomically distant dicot Populus (it was isolated from tobacco).

The deleterious effects of barnase observed in this study may have been exacerbated by growth in a field environment. No adverse effects on growth were observed in the greenhouse studies of oilseed rape (Mariani et al. 1990), tobacco (Mariani et al. 1990), or wheat (De Block et al. 1997). These plants showed normal height, leaf size, tillering, and/or high vigor based on causal observation. They also found no abnormalities in any of the flowering organs except for the tapetum. In the randomized greenhouse study on Arabidopsis and tobacco by Lemmetyinen et al. (2001), there were no differences in growth of transgenic vs. control lines until flowering began. Likewise, Wei et al. (2006) reported normal growth and morphology of greenhouse-grown trees expressing barnase under the poplar $L E A F Y$ promoter, whereas their field-grown transgenic trees had highly abnormal morphology and reduced growth. Surprisingly, this occurred in spite of coexpression of the barnase inhibitor barstar (Wei et al.
2006). In contrast, Zhang et al. (2012) did not observe any growth impairment in their field-grown, male-sterile pines and eucalypts, possibly because of the reduced toxicity of the barnase variant employed. They did not, however, present any data or statistical analysis in support of this observation. These results suggest that barnase toxicity can vary widely depending on species and growth environment. It also supports the need for steps to reduce barnase toxicity due to misexpression, which could include the use of spacers (Jagannath et al. 2001), separation of subunits among plants following crossing (Burgess et al. 2002; Bihao et al. 2012), or attenuated versions of the protein (Zhang et al. 2012).

The male sterility we observed was expressed over 4 years in the field. Similarly, Zhang et al. (2012) reported complete male sterility over 4 years in pine and over 2 years in eucalypts. Tobacco and silver birch transformed with the BpMADS1::BARNASE and the BpFULL1::BARNASE constructs were highly sterile for three and two consecutive years, respectively (Lännenpää et al. 2005). Commercial male-sterile Brassica has been authorized for use since 1996 in the USA and Canada (CERA 2013). Thus, it appears that barnase expression can be a highly reliable means for generating male sterility.

There remain a number of research needs if barnase technology is to become a general tool for transgene containment in forest trees. These include statistically robust and long-term field tests evaluating the level of sterility and impacts on vegetative growth and the development of new promoters that are more specific in their expression patterns and/or employ less toxic forms of cytotoxin genes. Female sterility will also be needed for many forest trees due to animal, wind, and water dispersal of seeds. This is particularly true for poplars, which 
have seeds that are very small and can "float" in air and on water over large distances. To our knowledge, no genes that cause bisexual or female sterility appear to have been fieldtested in any plant species.

Field tests are essential for determining the efficacy and stability of transgenic sterility. Unfortunately, the stringent regulation of all forms of direct genetic modification that are in place around the world makes even small field trials very difficult and, in many cases, impossible to carry out (Viswanath et al. 2012). The development of robust containment technology would therefore benefit not only from additional laboratory research, but from more discriminating regulatory systems that are based on trait risks and benefits, not on a presumed hazard due to use of recombinant DNA methods (Strauss et al. 2010; Meilan et al. 2012).

Acknowledgments We thank Plant Genetic Systems (Belgium) for providing the barnase construct for testing, Monsanto for providing the vector backbone for the reporter constructs, Jim Etherington for help in catkin harvesting, and Kathy Cook (Oregon State University) for conducting the anther histological studies.

Data archiving statement Data used in this manuscript will be made publicly available through DRYAD (http://datadryad.org/depositing).

Open Access This article is distributed under the terms of the Creative Commons Attribution License which permits any use, distribution, and reproduction in any medium, provided the original author(s) and the source are credited.

\section{References}

An Y, McDowell J, Huang S, McKinney E, Chambliss S, Meagher R (1996) Strong, constitutive expression of the Arabidopsis ACT2/ACT8 actin subclass in vegetative tissues. Plant J 10:107-121

Benfey P, Chua N (1990) The cauliflower mosaic virus 35 s promoter: combinatorial regulation of transcription in plants. 250:959-966

Bihao C, Xiaosan W, Jianjun L, Xiou X, Qinhua C (2012) Inducing male sterility of tomato using two component system. Plant Cell Tissue Organ 111:163-172

Brunner A, Li J, DiFazio S, Shevchenko O, Montgomery B, Mohamed R, Wei H, Ma C, Elias A, VanWormer K et al (2007) Genetic containment of forest plantations. Tree Genet Genomes 3:75-100

Burgess D, Ralston E, Hanson W, Heckert M, Ho M, Jenq T, Palys J, Tang K, Gutterson N (2002) A novel, two-component system for cell lethality and its use in engineering nuclear male-sterility in plants. Plant J 31:113-125

CERA (2013) GM Crop Database. Center for Environmental Risk Assessment. Washington D.C., USA. URL: http://www.cera-gmc. org/?action=gm_crop_database \&mode $=$ ShowProd\&data $=$ MS8xRF3

Cook S, Stanley R (1960) Tetrazolium chloride as an indicator of pine pollen germinability. Silvae Genet 9:134-136

Dalton D, Murthy G, Strauss S (2013) Production of traditional and novel biopolymers in transgenic woody plants. In: Gang DR (ed) Phytochemicals, plant growth, and the environment. Springer, New York, pp 59-78
De Block M, Debrouwer D, Moens T (1997) The development of a nuclear male sterility system in wheat. Expression of the barnase gene under the control of tapetum specific promoters. Theor Appl Genet 95:125-131

R Development Core Team (2013) R: a language and environment for statistical computing. R Foundation for Statistical Computing, Vienna, Austria. URL: http://www.R-project.org/

DiFazio S, Leonardi S, Slavov G, Garman S et al (2012) Gene flow and simulation of transgene dispersal from hybrid poplar plantations. New Phytol 193:903-915

Filichkin S, Meilan R, Busov V, Ma C, Brunner A, Strauss S (2006) Alcohol-inducible gene expression in transgenic Populus. Plant Cell Rep 25:660-667

Gilissen L, Metz P, Stiekema W, Nap J (1998) Biosafety of E. coli betaglucuronidase (GUS) in plants. Transgenic Res 7:157-163

Harfouche A, Meilan R, Altman A (2011) Tree genetic engineering and applications to sustainable forestry and biomass production. Trends Biotechnol 29:9-17

Hinchee M, Rottmann W, Mullinax L, Zhang C, Chang S, Cunningham M, Pearson L, Nehra N (2009) Short-rotation woody crops for bioenergy and biofuels applications. In Vitro Cell Dev Biol Plant 45:619-629

Horstmann V, Huether C, Jost W, Reski R, Decker E (2004) Quantitative promoter analysis in Physcomitrella patens: a set of plant vectors activating gene expression within three orders of magnitude. BMC Biotechnol 4:13

Hothorn T, Bretz F, Westfall P (2008) Simultaneous inference in general parametric models. Biom J 50:346-363

Huang S, An Y, McDowell J, McKinney E, Meagher R (1997) The Arabidopsis ACT11 actin gene is strongly expressed in tissues of the emerging inflorescence, pollen, and developing ovules. Plant Mol Biol 33:125-139

Jagannath A, Bandyopadhyay P, Arumugam N, Gupta V, Burma P, Pental D (2001) The use of a Spacer DNA fragment insulates the tissuespecific expression of a cytotoxic gene (barnase) and allows highfrequency generation of transgenic male sterile lines in Brassica juncea $\mathrm{L}$. Mol Breed 8:11-23

James R, DiFazio S, Brunner A, Strauss S (1998) Environmental effects of genetically engineered woody biomass crops. Biomass Bioenergy 14:403-414

Lännenpää M, Hassinen M, Ranki A, Hölttä-Vuori M, Lemmetyinen J, Keinonen K, Sopanen T (2005) Prevention of flower development in birch and other plants using a BpFULL1::BARNASE construct. Plant Cell Rep 24:69-78

Lemmetyinen J, Pennanen T, Lännenpää M, Sopanen T (2001) Prevention of flower formation in dicotyledons. Mol Breed 7:341-350

Lemmetyinen J, Keinonen K, Sopanen T (2004) Prevention of the flowering of a tree, silver birch. Mol Breed 13:243-249

Lenth R (2013) lsmeans: least-squares means. R package version 1.10-01

Li J, Brunner A, Shevchenko O, Meilan R, Ma C, Skinner J, Strauss S (2007) Efficient and stable transgene suppression via RNAi in fieldgrown poplars. Transgenic Res 17:679-694

Mariani C, Beuckeleer M, Truettner J, Leemans J, Goldberg R (1990) Induction of male sterility in plants by a chimaeric ribonuclease gene. Nature 347:737-741

Meilan R, Harfouche A, Nehra N (2012) Risk assessment of biotech trees: a path forward. J Forest 110:171-172

Miki B, McHugh S (2004) Selectable marker genes in transgenic plants: applications, alternatives and biosafety. J Biotechnol 107:193-232

Murashige T, Skoog F (1962) A revised medium for rapid growth and bio assays with tobacco tissue cultures. Physiol Plant 15:473-497

Ouakfaoui S, Miki B (2005) The stability of the Arabidopsis transcriptome in transgenic plants expressing the marker genes nptII and uidA. Plant J 41:791-800

Pinheiro J, Bates D, DebRoy S, Sarkar D, the R Development Core Team (2013) nlme: linear and nonlinear mixed effects models. R package version 3.1-111 
Rosellini D, Pezzotti M, Veronesi F (2001) Characterization of transgenic male sterility in alfalfa. Euphytica 118:313-319

Samac D, Tesfaye M, Dornbusch M, Saruul P, Temple S (2004) A comparison of constitutive promoters for expression of transgenes in alfalfa (Medicago sativa). Transgenic Res 13:349-361

Sanders P, Winter J, Barnason A, Rogers S, Fraley R (1987) Comparison of cauliflower mosaic virus $35 \mathrm{~S}$ and nopaline synthase promoters in transgenic plants. Nucleic Acids Res 15:1543-1558

Schneider C, Rasband W, Eliceiri K (2012) NIH Image to ImageJ: 25 years of image analysis. Nat Methods 9:671-675

Sheppard L, Brunner A, Krutovskii K, Rottmann W, Skinner J, Vollmer S, Strauss S (2000) A DEFICIENS homolog from the dioecious tree black cottonwood is expressed in female and male floral meristems of the two-whorled, unisexual flowers. Plant Physiol 124:627-640

Skinner J, Meilan R, Brunner A, Strauss S (2000) Options for genetic engineering of floral sterility in forest trees. In: Jain SM, Minocha SC (eds) Molecular biology of woody plants. Springer, Netherlands, pp 135-153

Skinner J, Meilan R, Ma C, Strauss S (2003) The Populus PTD promoter imparts floral-predominant expression and enables high levels of floral-organ ablation in Populus, Nicotiana and Arabidopsis. Mol Breed 12:119-132

Slavov G, Leonardi S, Burczyk J, Adams W, Strauss S, Difazio S (2009) Extensive pollen flow in two ecologically contrasting populations of Populus trichocarpa. Mol Ecol 18:357-373

Strauss S, Schmitt M, Sedjo R (2009a) Forest scientist views of regulatory obstacles to research and development of transgenic forest biotechnology. J For 107:350-357
Strauss S, Tan H, Boerjan W, Sedjo R (2009b) Strangled at birth? Forest biotech and the convention on biological diversity. Nat Biotechnol 27:519-527

Strauss S, Kershen D, Bouton J, Redick T, Tan H, Sedjo R (2010) Far-reaching deleterious impacts of regulations on research and environmental studies of recombinant DNA-modified perennial biofuel crops in the United States. Bioscience 60:729741

Ulyanova V, Vershinina V, Ilinskaya O (2011) Barnase and binase: twins with distinct fates. FEBS J 278:3633-3643

Vining K, Contreras R, Ranik M, Strauss S (2012) Genetic methods for mitigating invasiveness of woody ornamental plants: research needs and opportunities. HortSci 47:1210-1216

Viswanath V, Albrectsen B, Strauss S (2012) Global regulatory burden for field testing of genetically modified trees. Tree Genet Genomes 8:221-226

Wei H, Meilan R, Brunner A, Skinner J, Ma C, Gandhi H, Strauss S (2006) Field trial detects incomplete barstar attenuation of vegetative cytotoxicity in Populus trees containing a poplar LEAFY promoter::barnase sterility transgene. Mol Breed 19:69-85

Yoo S, Bomblies K, Yoo S, Yang J, Choi M, Lee J, Weigel D, Ahn J (2005) The 35S promoter used in a selectable marker gene of a plant transformation vector affects the expression of the transgene. Planta 221:523-530

Zhang C, Norris-Caneda K, Rottmann W, Gulledge J, Chang S, Kwan B, Thomas A, Mandel L, Kothera R, Victor A et al (2012) Control of pollen-mediated gene flow in transgenic trees. Plant Physiol 159: 1319-1334 